\title{
Double dissociation of working memory load effects induced by bilateral parietal modulation
}

\author{
Marco Sandrini a,b,*, Anna Fertonani ${ }^{\mathrm{c}}$, Leonardo G. Cohen ${ }^{\mathrm{a}}$, Carlo Miniussi ${ }^{\mathrm{c}, \mathrm{d}}$ \\ ${ }^{a}$ Human Cortical Physiology and Stroke Neurorehabilitation Section, National Institute of Neurological Disorders and Stroke, National Institutes of Health, Bethesda, MD, USA \\ ${ }^{\mathrm{b}}$ Center for Neuroscience and Regenerative Medicine at Uniformed Services University of Health Sciences, Bethesda, MD, USA \\ ${ }^{c}$ Cognitive Neuroscience Section, IRCCS Centro San Giovanni di Dio Fatebenefratelli, Brescia, Italy \\ d Department of Biomedical Sciences and Biotechnologies, National Institute of Neuroscience, University of Brescia, Brescia, Italy
}

\section{A R T I C L E I N F O}

\section{Article history:}

Received 1 June 2011

Received in revised form

21 November 2011

Accepted 18 December 2011

Available online 27 December 2011

\section{Keywords:}

Transcranial direct current stimulation

Parietal lobe

Working memory

n-Back task

Familiarity

\begin{abstract}
A B S T R A C T
Transcranial magnetic stimulation and neuroimaging data have revealed bilateral posterior parietal cortex (PPC) involvement during verbal $n$-back working memory (WM). In this task as $n$ (i.e., WM load) increases, subjects show poorer behavioral performance as well as greater activation of this brain area. Moreover, there is evidence that a brief period of practice or even increased familiarity with the task can improve WM performance and lead to activation changes in the PPC. The aim of this study was to investigate, using transcranial direct current stimulation (tDCS), the effects on WM load performance induced by different PPC modulation after increased familiarity with the task. After a short practice, we tested verbal WM using an $n$-back task (1-back vs. 2-back) before and after the application of bilateral tDCS over PPCs (left anodal-right cathodal, left cathodal-right anodal or sham). ANOVA showed a significant interaction between tDCS and task. In the 1-back task, left anodal-right cathodal modulation abolished improvement in reaction times observed in the other two modulation conditions. Conversely, in the 2-back task the same effect was observed after left cathodal-right anodal modulation relative to the other two modulation conditions. This double dissociation demonstrates either a differential engagement of each PPC or changes in the interhemispheric balance of activity across this brain region. Neuroimaging studies show parametric activation of the PPC as difficulty increases, but activation does not switch sides. Thus, our observed effects cannot be attributed to increased task difficulty, the stimuli used, or the response requirements. Rather, we suggest that these findings reflect the use of different processing strategies to perform these two tasks. In conclusion, after increased familiarity with the task, different tDCS modulations lead to changes in a task-related region depending on differences in processing strategies in 1-back vs. 2-back.
\end{abstract}

(c) 2011 Elsevier Ltd. All rights reserved.

\section{Introduction}

It has been hypothesised that higher brain functions such as language, planning and problem solving rely on working memory (WM) i.e., a system that acts to temporarily maintain and manipulate task-relevant information (Baddeley, 1986; Just \& Carpenter, 1992; Shallice, 1988). Several theories on WM have been proposed (for review see Miyake \& Shah, 1999). At present the model proposed by Baddeley and Hitch (1974) is one of the most extensively investigated theoretical constructs of WM. The authors proposed the existence of three functional components of WM. A central

\footnotetext{
* Corresponding author at: Human Cortical Physiology and Stroke Neurorehabilitation Section, National Institute of Neurological Disorders and Stroke, NIH. Building 10, Room 7D52, Bethesda, MD 20892, USA. Tel.: +1 301496 9986; fax: +1 3014027010

E-mail address: marco.sandrini@nih.gov (M. Sandrini).
}

executive was envisioned as a control system of limited attentional capacity that is responsible for the manipulation of information within WM and for controlling two subsidiary storage systems: a phonological loop, which is based on sound and language, and a visuospatial sketchpad. The phonological loop was assumed to be responsible for the storage and maintenance of information in a phonological form and consists of two parts: a short-term phonological store and an articulatory rehearsal component that can revive the memory trace. The visuospatial sketchpad was dedicated instead to the storage and maintenance of visual and spatial information. Based on a number of empirical findings a fourth component, the episodic buffer, was added (Baddeley, 2000). The episodic buffer is assumed to be a limited capacity store that is capable of multi-dimensional coding to allow the binding of information to create integrated episodes.

Regarding the neural substrates of WM, the dorsolateral prefrontal cortex (DLPFC) has been shown to be related to central executive processes (for review see Smith \& Jonides, 1998). While 
Broca's area has been associated with the articulatory rehearsal component, the posterior parietal cortex (PPC) has been claimed to be the site that mediates the storage systems: the phonological loop and the visuospatial sketchpad (Baddeley, 2003; Jonides, Schumacher, et al., 1998; Smith, Jonides, Marshuetz, \& Koeppe, 1998; Todd \& Marois, 2004; Xu \& Chun, 2006, but see Buchsbaum \& D'Esposito, 2008 about the phonological loop). However, there is evidence that the functional neuroanatomy of WM may be more complex and anatomically distributed, with PPC playing a role also in the executive component (Cohen et al., 1997; Collette, Hogge, Salmon, \& Van der Linden, 2006; Mottaghy, Doring, Muller-Gartner, Topper, \& Krause, 2002).

A common task used to study WM is the " $n$-back" (Gevins \& Cutillo, 1993), a task that has been designed to manipulate factors associated with WM such as load (Carter et al., 1998). In the most typical variant of this task, the participant is required to monitor a series of stimuli (e.g., letters) presented centrally and to respond whenever a stimulus that is the same as the one presented $n$ trials previously is presented, where $n$ is a pre-specified integer (usually 1, 2, or 3). As $n$ increases, there is a greater demand on WM and consequently a poorer behavioral performance. Therefore, this task requires the simultaneous engagement of several retention- and control-related operations and is therefore assumed to place great demands on a number of key processes within WM.

In recent years, variants of the n-back procedure have been employed to investigate the neural basis of WM processes. Neuroimaging (Owen, McMillan, Laird, \& Bullmore, 2005) and transcranial magnetic stimulation (Mottaghy et al., 2002; Mottaghy, Gangitano, Krause, \& Pascual-Leone, 2003; Mottaghy, PascualLeone, et al., 2003) studies have shown that in addition to the contribution of prefrontal cortex (PFC), n-back studies have frequently demonstrated the involvement of PPC regions, particularly the superior and inferior parietal lobes (SPL, and IPL, respectively) (for reviews see Cabeza \& Nyberg, 2000; Mottaghy, 2006; Owen et al., 2005).

Regarding the WM load, neuroimaging studies have reported frontal and parietal activity increases during 2-back relative to 1-back task performance as well as parametric variations of $n$. Specifically, the activated regions are common and activation does not switch sides with increasing difficulty (Braver et al., 1997; Cohen et al., 1997; Jonides et al., 1997a; Ragland et al., 2002). This increase in activation presumably reflects the augmented load on control processes devoted to holding items and temporal information in WM, and transient 'updating' of maintained representations (Smith \& Jonides, 1999). Updating during the 2-back task constitutes several additional subcomponents that become more critical relative to 1-back, such as temporal coding and eliminating selective information from WM (Jonides et al., 1997b; Postle, Berger, Goldstein, Curtis, \& D'Esposito, 2001). Inhibition, monitoring and selection processes should be crucial for the updating function and for keeping track of item order, but also in the implementation of interference resolution during source judgments involving recent context irrelevant items (e.g., items presented "2-back" in the 1back task) (Badre \& Wagner, 2005; D’Esposito, Postle, Jonides, \& Smith, 1999; Jonides, Smith, Marshuetz, Koeppe, \& Reuter-Lorenz, 1998).

Regarding the laterality, verbal n-back tasks appear to activate a fronto-parietal network in the left hemisphere while spatial n-back tasks appear to activate a right hemisphere network (D'Esposito et al., 1998; Fiez et al., 1996), although this pattern is by no means unequivocal and bilateral activity often occurs in both type of tasks (Braver et al., 1997; Nystrom et al., 2000). For example, neuroimaging studies during verbal $n$-back have reported bilateral activation of PPC. While some researchers have attributed right parietal activity to the necessity of spatial processing in some verbal WM tasks
(Clark et al., 2000; Honey, Bullmore, \& Sharma, 2000; Salmon et al., 1996) others have attributed more domain-general functions to the right parietal lobe, such as selective attention, that may be required when performing verbal WM tasks (Chein, Ravizza, \& Fiez, 2003; Jonides, Schumacher, et al., 1998; LaBar, Gitelman, Parrish, \& Mesulam, 1999). Both domain-specific (i.e., spatial coding) and domain-general accounts of right parietal contributions to verbal WM are supported by the literature making it difficult to differentiate between them.

Moreover, there is evidence that 1-back task and 2 or 3-back task do not just differ in difficulty, but also in the strategies used to perform them. Specifically, it has been shown that during a verbal n-back task, subjects might use an "activation" strategy, in which they respond to each letter based on its familiarity, or an "update" strategy, in which more WM resources are involved because they have to actively maintain a list of the prior letters and update that list after each letter is presented (Lovett, Daily, \& Reder, 2000). Recognition memory can be supported by both an undifferentiated, strength-like memory signal (usually referred to as familiarity), and by the retrieval of qualitative information about the episode such as contextual details (usually referred to as recollection) (Yonelinas, 2002). Performance in the n-back task is consistent with this familiarity and recollection account, in which 1-back task is based on familiarity while 2 or 3-back tasks are based more on recollection (Harbison, Atkins, \& Dougherty, 2011). Regarding the neural substrates of these processes, there is evidence that they are characterized by different patterns of brain activity in frontal, parietal and medial temporal cortices (Cabeza, Ciaramelli, Olson, \& Moscovitch, 2008; Diana, Yonelinas, \& Ranganath, 2007; Duarte, Ranganath, Winward, Hayward, \& Knight, 2004; Skinner \& Fernandes, 2007; Vilberg \& Rugg, 2008). Additionally, there are findings showing that recollective memories are frequently observed more in the left hemisphere while familiarity based-traces more in the right hemisphere (Dobbins, Simons, \& Schacter, 2004; Duarte, Ranganath, \& Knight, 2005; Henson, Rugg, Shallice, Josephs, \& Dolan, 1999; Kensinger, Clarke, \& Corkin, 2003; Mitchell, Johnson, Raye, \& Greene, 2004; Nolde, Johnson, \& D’Esposito, 1998; Vilberg \& Rugg, 2009).

Finally, a brief period of practice with a WM task can improve performance and modify underlying patterns of neural activation (Kelly \& Garavan, 2005). Whereas some studies have reported an increased activation in the PPC following a small amount of practice on WM tasks (Kirschen, Chen, Schraedley-Desmond, \& Desmond, 2005), others have found decreased activation (Garavan, Kelley, Rosen, Rao, \& Stein, 2000). Furthermore, even increased familiarity with the task can improve WM performance and lead to activation changes in the PPC (Jolles, Grol, Van Buchem, Rombouts, \& Crone, 2010).

The aim of this study was to investigate, using bilateral transcranial direct current stimulation (tDCS), the effects on WM load performance induced by different PPC modulations after increased familiarity with the task.

tDCS is a non-invasive technique for modulating cortical excitability by constantly applying weak electrical current over time to enhance (anodal modulation) or reduce (cathodal modulation) the excitation of neuronal populations, with a maximal effect on the stimulated area beneath the electrodes (Nitsche et al., 2008; Priori, 2003; Utz, Dimova, Oppenlander, \& Kerkhoff, 2010).

After a short period of time in which subjects practiced with the task to increase familiarity, we tested verbal WM using a verbal nback task (1-back vs. 2-back). WM was tested before and after the application of bilateral tDCS over PPCs (left anodal-right cathodal, left cathodal-right anodal and sham) in a between-subject design. The application of this electrode montage allowed us to modulate activity in the left and right PPC in opposite directions simultaneously. 
On the basis of lateralization findings described above, we hypothesized that in the 1-back task, left anodal-right cathodal modulation would abolish the improvement in reaction times compared to the other two modulation conditions. Conversely, in the 2-back task the same effect would be observed after left cathodalright anodal modulation relative to the other two modulation conditions.

\section{Methods}

\subsection{Subjects}

Twenty-seven healthy subjects were randomly assigned to one of three groups (five males and four females in each group, mean age $25 \pm 2$ years, range 20-30). Two of the groups received active modulation, and one received sham modulation. All subjects were right-handed (mean score on the Edinburgh Handedness Inventory $=92.9$ ), with normal or corrected-to-normal vision. Exclusion criteria included a history of seizures, implanted metal objects, heart problems or any neurological disease. The study was approved by the Ethics Committee of IRCCS San Giovanni di Dio, Fatebenefratelli, Brescia, Italy.

\subsection{Experimental tasks}

In the $n$-back task, subjects were shown a continuous sequence of stimuli. For each stimulus, the subjects had to decide whether it matched the stimulus shown n stimuli earlier in the sequence (Gevins \& Cutillo, 1993). In the 1-back condition, the target was any stimulus that was identical to the one immediately preceding it (i.e., one trial prior). In the 2-back condition, the target was any stimulus that was identical to the one presented two trials prior. Therefore, for each trial, subjects had to: (1) maintain the current stimulus and the last two stimuli in WM, (2) evaluate the current stimulus with the $n-2$ stimulus, (3) answer 'yes' or 'no', (4) dump the $n-2$ stimulus, and (5) continue maintaining the $n-1$ and $n$ stimuli for the next trial. It is clear that this task requires wide manipulation of information (e.g., updating, temporal ordering, and inhibition processes) rather than simple selection and maintenance of information.

Letters were presented centrally in 40-point Arial font, randomly presented in upper or lowercase. Subjects were told not to distinguish between upper and lowercase presentations of the same letter. This mixing of cases was intended to encourage subjects to encode and rehearse letter stimuli as verbal phonemes instead of as visual letter forms. The letter stimuli were chosen from a set of 16 consonants letters (all the consonants except J, W, and Y). The stimuli were presented on a $17 \mathrm{in}$. monitor as white against a grey background.

The experiment was controlled by a PC running the Superlab Pro software (Cedrus Corporation, San Pedro, CA, USA), version 2.0.4. Participants were seated facing a computer monitor at a distance of $70 \mathrm{~cm}$. Each test began with the relative instruction for a 1-back or 2-back task, followed by 36 stimuli. Matches occurred in $33 \%$ of the trials. Stimuli were presented for 250 ms every $3000 \mathrm{~ms}$ (interstimulus interval). A fixation point appeared in the centre of the screen $1000 \mathrm{~ms}$ before the stimulus. Before the beginning of the experiment, subjects were trained in a block of 54 stimuli for the 2-back task. Subjects were included in the study only after reaching a criterion level of performance ( $>75 \%$ accuracy). Participants were instructed to respond with both hands (index finger) by pressing one of two buttons (e.g., left finger, target stimuli; right finger, non-target stimuli) on the keyboard as quickly as possible after the presentation of the stimulus. The assignment of the response buttons and the order of execution of the two tasks were counterbalanced across subjects.

\subsection{Transcranial direct current stimulation}

The modulation was delivered by a battery-driven constant current stimulator (Eldith-Plus, neuro-Conn GmbH, Ilmenau, Germany) through a pair of saline-soaked sponge electrodes $(5 \mathrm{~cm} \times 7 \mathrm{~cm})$. A constant current of $1.5 \mathrm{~mA}$ was applied for $13 \mathrm{~min}$. The current density $\left(42.86 \mu \mathrm{A} / \mathrm{cm}^{2}\right)$ was maintained below the safety limits (Poreisz, Boros, Antal, \& Paulus, 2007). The electrodes were held firmly by elastic bands, and an electroconductive gel was applied under the electrodes before the test to reduce skin impedance. The electrodes were placed on the left and right PPC, respectively centred over P3 - P4 according to the 10-20 EEG international electrode scalp positioning system (Jasper, 1958).

Although we can determine with precision that the centre of electrodes was placed over P3 and P4 (intraparietal sulcus, Brodmann area 7/40, see Herwig, Satrapi, \& Schonfeldt-Lecuona, 2003), we do not know the precise parietal areas that are directly influenced by the electric field. Therefore, we can only assume that we were stimulating the PPC underlying the electrode.

With this montage it is possible to modulate activity in the left and right PPC in opposite directions simultaneously. The placement of the electrodes over both parietal lobes increased the specificity of the type of modulation, avoiding indirect modulation of other areas with the reference electrode. The bilateral montage allows better control of the investigated variables, because it allows the equal spreading of a modulation and thus equal intensity (although in the opposite direction) on the two hemispheres. Conversely, cephalic unilateral montages (with one electrode on the target brain region and the other on a region, sometimes erroneously, assumed not to be involved in the investigated processes) can result in uncontrolled effects simply linked to the inadequate positioning of the reference electrode.

The selective influence of tDCS on different cognitive processes after the application of a bilateral montage have already been demonstrated in the primary motor cortex (Vines, Cerruti, \& Schlaug, 2008), PFC (Boggio et al., 2010; Fecteau et al. 2007; Hecht, Walsh, \& Lavidor, 2010), anterior temporal lobe (Boggio et al., 2009; Chi, Fregni, \& Snyder, 2010), frontotemporal region (Penolazzi et al., 2010) and PPC (Cohen Kadosh, Soskic, Iuculano, Kanai, \& Walsh, 2010).

We applied three bilateral parietal tDCS modulations in the three groups: anoda over left hemisphere and cathodal over right hemisphere (referred to as LHA-RHC) in the first group, cathodal over left hemisphere and anodal over right hemisphere (referred to as LHC-RHA) in the second group and sham modulation (referred to as SHAM) in the third group (see Fig. 1).

For SHAM modulation, the current was turned off $30 \mathrm{~s}$ after the beginning of the modulation. Thus, the subjects felt the itching sensation below the electrodes at the beginning of the modulation that was indistinguishable from the real one (Gandiga Hummel, \& Cohen, 2006)

In each group, after a short practice with the tasks, SHAM was always the first type of modulation, followed by verbal WM tasks (referred to as "pre" modulation). After $10 \mathrm{~min}$ of rest, there was a second period of modulation (active or sham), followed by another presentation of the WM tasks (referred to as "post" modulation) (see Fig. 1). The tasks lasted about $5 \mathrm{~min}$ and considering that the duration of the excitability changes induced by tDCS are stable for up to about an hour if tDCS is applied for 9-13 min (Nitsche et al., 2008), we can be sure that the effects of tDCS cover the whole time.

\section{Results}

All subjects tolerated the modulation well and none asked to stop the experiment.

Analysis was conducted on reaction times (RTs) and error rates. Data from single trials with RTs that fell below or above two standard deviations (SD) from each individual's average were eliminated. This procedure eliminated $0.9 \%$ of responses for 1 -back task, $1.2 \%$ for 2 -back task. Furthermore incorrect responses were discarded. Post hoc comparisons were made with two-tailed independent $t$-tests, and the $P$-value was Bonferroni-corrected for the number of comparisons and set at $p=0.016$.

\subsection{Error rate}

The percentage of errors was very low (mean $3.1 \%$ for 1 -back and $3.6 \%$ for 2-back, range $2.4-5.1 \%$ ). The mean value of tasks, time and $t D C S$ modulations is given in Table $1 \mathrm{a}$. We performed a twofactorial ANOVA for repeated measures in which the dependent variable was the error rates with a $3 \times 2 \times 2$ factorial design: $t D C S$ (LHA-RHC, LHC-RHA, and SHAM) as the between-subject factor and task (1-back vs. 2-back) and time (pre vs. post) as withinsubject factors. The main effect $\operatorname{tDCS}\left[F_{(2 ; 24)}=0.437 ; p=0.651\right]$ task $\left[F_{(1 ; 24)}=1.304 ; p=0.265\right]$, time $\left[F_{(1 ; 24)}=1.141 ; p=0.296\right]$, and the interactions task $x$ time $\left[F_{(2 ; 24)}=0.681 ; p=0.417\right], t D C S \times$ task $\left[F_{(2 ; 24)}=0.202 ; p=0.818\right], \mathrm{tDCS} \times$ time $\left[F_{(2 ; 24)}=0.318 ; p=0.731\right]$ and $t D C S \times$ task $\times$ time $\left[F_{(2 ; 24)}=0.497 ; p=0.614\right]$ were all not statistically significant.

\subsection{Reaction times}

The mean value of tasks, time and tDCS modulations is given in Table 1b. Initially, we performed a two-factorial ANOVA for repeated measures in which the dependent variable was RTs at pre-modulation with a $3 \times 2$ factorial design: $t D C S$ (LHA-RHC, LHC-RHA, and SHAM) as the between-subject factor and task (1-back vs. 2-back) as the within-subject factor. The main effect task was significant $\left[F_{(1 ; 24)}=46.5 ; p<0.01\right]$ (1-back faster than 2-back), but the main effect $t D C S$ and the interaction $t D C S x$ task were not significant $\left[F_{(2 ; 24)}=0.316 ; p=0.73\right.$ and $F_{(2 ; 24)}=0.083$; $p=0.92$, respectively]. Therefore, the ANOVA revealed that there 


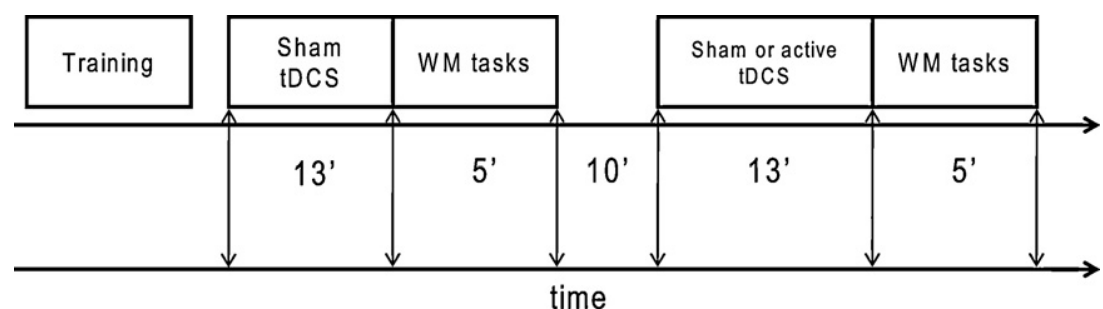

Fig. 1. The different phases of the experimental paradigm are reported along the time line.

Table 1

Error rate (1a) and RTs (1b) of tasks pre and post the three tDCS conditions (SHAM, LHA-RHC, and LHC-RHA). Data are presented as mean (Standard Error).

\begin{tabular}{|c|c|c|c|c|c|c|}
\hline \multicolumn{7}{|l|}{ (a) } \\
\hline \multirow[t]{3}{*}{ Error rate \% } & \multicolumn{2}{|l|}{ SHAM } & \multicolumn{2}{|l|}{ LHA-RHC } & \multicolumn{2}{|l|}{ LHC-RHA } \\
\hline & Pre & Post & Pre & Post & Pre & Post \\
\hline & Mean (SE) & Mean (SE) & Mean (SE) & Mean (SE) & Mean (SE) & Mean (SE) \\
\hline 1-Back task & $3.0(0.7)$ & $2.7(1.2)$ & $2.4(0.7)$ & $3.3(1.2)$ & $3.6(0.7)$ & $3.3(1.2)$ \\
\hline 2-Back task & $3.3(0.9)$ & $3.6(1.1)$ & $2.7(0.9)$ & $3.3(1.1)$ & $3.6(0.9)$ & $5.1(1.1)$ \\
\hline \multicolumn{7}{|l|}{ (b) } \\
\hline \multirow[t]{3}{*}{ RTs ms } & \multicolumn{2}{|l|}{ SHAM } & \multicolumn{2}{|l|}{ LHA-RHC } & \multicolumn{2}{|l|}{ LHC-RHA } \\
\hline & Pre & Post & Pre & Post & Pre & Post \\
\hline & Mean (SE) & Mean (SE) & Mean (SE) & Mean (SE) & Mean (SE) & Mean (SE) \\
\hline 1-Back task & $602(20)$ & $547(18)$ & $608(18)$ & $612(23)$ & $582(30)$ & $524(31)$ \\
\hline 2-Back task & $691(13)$ & $623(14)$ & $683(11)$ & $628(18)$ & $674(22)$ & $677(23)$ \\
\hline
\end{tabular}

was no difference between the three tDCS conditions in the pre-modulation (baseline) phase for both tasks.

We then performed a two-factorial ANOVA for repeated measures in which the dependent variable was delta RTs (post modulation-pre modulation) with a $3 \times 2$ factorial design: tDCS (LHA-RHC, LHC-RHA, and SHAM) as the between-subject factor and task (1-back vs. 2-back) as the within-subject factor. The main factor task was not significant $\left[F_{(1 ; 24)}=0.079 ; p=0.78\right]$, whereas the main factor $t D C S\left[F_{(2 ; 24)}=4.33 ; p<0.05\right.$; sham $=-61$, LHA-RHC $=-26$, LHC-RHA $=-25]$ and the interaction $t D C S \times$ task $\left[F_{(2 ; 24)}=15.2\right.$; $p<0.01$ ] were significant.

Direct post hoc comparison ( $t$-test) showed significant differences in the 1-back task between LHA-RHC and SHAM $(p=0.001)$, between LHC-RHA and LHA-RHC $(p=0.004)$ but not between SHAM and LHC-RHA ( $p=0.849$ ). In contrast, in the 2-back task, there were significant differences between LHC-RHA and SHAM $(p=0.008)$, between LHA-RHC and LHC-RHA $(p=0.013)$ but not between SHAM and LHA-RHC $(p=0.530)$. Finally, regarding the effect directions, tDCS abolished the practice-dependent improvement in RTs in WM in both tasks (see Fig. 2).

\section{Discussion}

This study showed for the first time a double-dissociation of verbal WM load effects induced by differential PPC modulation after increased familiarity with the task.

Although bilateral tDCS abolished the improvement in RTs, this effect was induced by different tDCS modulation conditions in the 1-back and 2-back task. In the 1-back task LHA-RHC modulation abolished improvement in RTs observed in the other two modulation conditions. Conversely, in the 2-back task LHC-RHA modulation abolished the same effect relative to the other two modulation conditions.

Neuroimaging studies show parametric activation of the PPC as difficult increases, but activation does not switch sides. Therefore, this double-dissociation cannot be attributed to increased task difficulty, the stimuli used, or the response requirements. Rather, we propose that it reflects the use of different processing strategies to perform 1-back and 2-back. In order to accomplish these tasks, subjects might have used the "activation" strategy, which is based on familiarity, in the 1-back task, and might have shifted

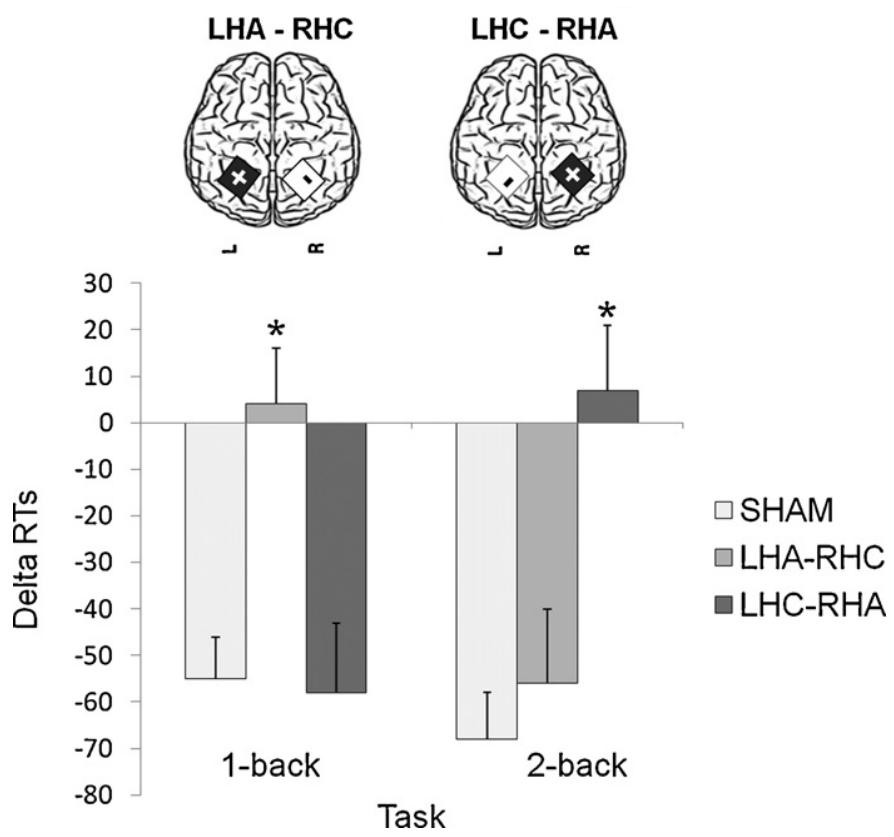

Fig. 2. Effects of tDCS on delta RTs (post modulation-pre modulation) across tDCS conditions and tasks. In the 1-back task, tDCS abolished the practice-dependent improvement in RTs after LHA-RHC modulation, whereas in the 2-back task, tDCS abolished the practice effect after LHC-RHA modulation. Vertical bars represent standard error; ${ }^{*} p<0.016$. On the graph, a schematic representation depicts the electrode montage over the left and right posterior parietal cortex (P3 and P4) using cathodal $(-)$ and anodal $(+)$ modulation. 
to the "update" strategy, which is based more on recollection, in the 2-back task (Lovett et al., 2000). Indeed, evidence of hemispheric specialization for strategic processing during WM has been already observed (Manoach et al., 2004). In this study, compared to shape WM, spatial WM performance was faster and more accurate and was associated with increased right ventrolateral PFC (VLPFC) activation. In contrast, compared to spatial WM, shape WM was associated with increased left VLPFC activity. In order to explain the increased activity in right PFC for spatial WM, the authors proposed the use of configural processing strategies for spatial WM. Therefore these findings, like ours, suggest that the differences in performance and in laterality might reflect the involvement of strategic differences in the two tasks.

Regarding the direction of behavioral performance, our bilateral modulation abolished the improvement in RTs in both WM tasks. Whereas some studies investigating verbal WM by means of tDCS found that anodal modulation over the left PFC enhances performance (Andrews, Hoy, Enticott, Daskalakis, \& Fitzgerald, 2011; Fregni et al., 2005; Ohn et al., 2008), other studies report conflicting results. For instance, an interference effect on verbal WM performance was reported using cathodal modulation over the left PFC (Elmer, Burkard, Renz, Meyer, \& Jancke, 2009), after intermittent tDCS over bilateral PFC (Marshall, Molle, Siebner, \& Born, 2005) and on visual WM after unilateral cathodal modulation over the right PPC (Berryhill, Wencil, Branch Coslett, \& Olson, 2010). Finally, similar to our data, Ferrucci et al. (2008) reported that bilateral anodal or cathodal tDCS over the cerebellum specifically impaired the practice-dependent proficiency increase in verbal WM (Sternberg test). Therefore, in addition to the polarity of modulation, effects of tDCS on WM often depend on various factors, such as the task, current density, modulation duration, electrode montage, electrode size and orientation of the electric field in relation to anatomical and geometrical feature of the cortex.

Our findings are also consistent with TMS studies that have shown the crucial role of PPC in simple short-term retention and in the manipulation of information in WM (Hamidi, Tononi, \& Postle, 2008; Koch et al., 2005; Postle et al., 2006) and with functional magnetic resonance imaging (fMRI) data showing changes in activation in the PPC and improved behavioral WM performance after increased familiarity with the task (Jolles et al., 2010). However, it should be noted also that the effects of tDCS on remote brain regions must be taken into account when tDCS of a specific cortical area is used to modulate cognitive functions. Many evidences support for the notion that tDCS applied over a specific cortical region induces widespread changes of cerebral activity at cortical and subcortical levels and alters functional connectivity between brain regions (Antal, Polania, Schmidt-Samoa, Dechent, \& Paulus, 2011; Keeser et al., 2011; Lang et al., 2005). Although the functional relevance of these distant changes must be shown, we cannot exclude the possibility that our bilateral electrode montage induced a modulation of activity in the fronto-parietal networks. Indeed, using path analysis of fMRI time series data, Honey et al. (2002) demonstrated that manipulation of cognitive demand in a verbal WM task (1 vs. 2-back) is associated with concomitant changes in the integrated function of the fronto-parietal networks. Specifically, the positive connection between the left inferior frontal gyrus and the left PPC was consistent across load conditions. However, at higher load, there was additionally a significant connection from the left middle frontal gyrus (MFG) to the left PPC via the supplementary motor area and enhanced connectivity between the right MFG and the left hemispheric frontoparietal regions. These findings provide evidence that the functional dissociation related to load and concomitant executive demand, as already described for PFC areas, may also be observable in an extended fronto-parietal network.
All these factors suggest that before clear conclusions can be drawn, some limitations must be kept in mind and investigated in future studies.

The first limitation is that the effect of tDCS is not particularly focused (Nitsche et al., 2008) and, thus, it is difficult to make definitive conclusions regarding neuroanatomical function. It is possible that different areas adjacent to or inside the PPC were modulated and we cannot define the contribution to our results of regions that have been shown to be important for verbal WM, such as the IPL (Baddeley, 2003), SPL (Koenigs, Barbey, Postle, \& Grafman, 2009; Postle et al., 2006) or the superior temporal cortex (Buchsbaum \& D’Esposito, 2008).

The second limitation is that we do not know whether the observed double-dissociation was primarily due to either changes in the interhemispheric balance of activity across the PPCs or considering the effect on the behavioral performance, due to cathodal modulation (i.e., right PPC in the 1-back and left PPC in the 2back). Nevertheless, this limitation may not be solved simply by including unilateral modulation groups in the study, partly because a monopolar electrode on one hemisphere may still affect the other hemisphere through current dispersion (Lang et al., 2005). It is worth mentioning here that progress in neuroscience often depends on the convergence of evidence from multiple methods. Since every single technique has its own limitations, there is a clear theoretical advantage in combining different approaches (Siebner et al., 2009). Therefore in future investigations, it may be worthwhile to explore other methods of modulation, such as unilateral modulation (i.e., extracephalic montage), in combination with neuroimaging techniques to investigate specific questions about the mechanisms of action leading to this double dissociation. Moreover, studies combining tDCS and neuroimaging techniques are needed to systematically investigate specific changes in brain network dynamics (for e.g., using graph theoretical approaches, Sporns, 2010 ) induced by tDCS, in order to provide a plausible mechanistic account of neuroplasticity and explain behavioral neurophysiological changes that are modulated by this modulation technique (Venkatakrishnan \& Sandrini, 2012).

In conclusion, we were able to demonstrate a double dissociation of WM load effects induced by bilateral parietal modulation after increased familiarity with the tasks. In the 1-back task, left anodal-right cathodal modulation abolished improvement in RTs observed in the other two modulation conditions. Conversely, in the 2-back task, left cathodal-right anodal modulation abolished the same effect relative to the other two modulation conditions.

This double dissociation demonstrates either a differential engagement of each PPC or changes in the interhemispheric balance of activity across this brain region. Thus, these effects cannot be attributed to increased task difficulty, the stimuli used, or the response requirements. Rather, we suggest that these findings reflect the use of different processing strategies to perform these two tasks. In this study we have shown that, after increased familiarity with the task, different tDCS modulations lead to changes in a task-related region depending on differences in processing strategies in 1-back vs. 2-back.

\section{Acknowledgments}

This work was supported by a NIH-Regione Lombardia (Italy) research career transition award (Regional provision n. 3472, 8th April 2008) to MS.

We would like to thank Anusha Venkatakrishnan and two anonymous reviewers for insightful comments and suggestions.

\section{References}

Andrews, S. C., Hoy, K. E., Enticott, P. G., Daskalakis, Z. J., \& Fitzgerald, P. B. (2011). Improving working memory: The effect of combining cognitive activity and 
anodal transcranial direct current stimulation to the left dorsolateral prefronta cortex. Brain Stimulation, 4(2), 84-89.

Antal, A., Polania, R., Schmidt-Samoa, C., Dechent, P., \& Paulus, W. (2011). Transcranial direct current stimulation over the primary motor cortex during fMRI. Neuroimage, 55(2), 590-596.

Baddeley, A. (1986). Working memory. New York: Oxford University Press.

Baddeley, A. (2000). The episodic buffer: A new component of working memory? Trends in Cognitive Sciences, 4(11), 417-423.

Baddeley, A. (2003). Working memory: Looking back and looking forward. Nature Reviews Neuroscience, 4(10), 829-839.

Baddeley, A., \& Hitch, G. (1974). Working memory. The psychology of learning and motivation: Advances in research and theory New York: Academic Press., pp. 47-89.

Badre, D., \& Wagner, A. D. (2005). Frontal lobe mechanisms that resolve proactive interference. Cerebral Cortex, 15(12), 2003-2012.

Berryhill, M. E., Wencil, E. B., Branch Coslett, H., \& Olson, I. R. (2010). A selective working memory impairment after transcranial direct current stimulation to the right parietal lobe. Neuroscience Letters, 479(3), 312-316.

Boggio, P. S., Campanha, C., Valasek, C. A., Fecteau, S., Pascual-Leone, A., \& Fregni, F. (2010). Modulation of decision-making in a gambling task in older adults with transcranial direct current stimulation. European Journal of Neuroscience, 31(3), 593-597.

Boggio, P. S., Fregni, F., Valasek, C., Ellwood, S., Chi, R., Gallate, J., et al. (2009). Temporal lobe cortical electrical stimulation during the encoding and retrieval phase reduces false memories. PLoS One, 4(3), e4959.

Braver, T. S., Cohen, J. D., Nystrom, L. E., Jonides, J., Smith, E. E., \& Noll, D. C. (1997). A parametric study of prefrontal cortex involvement in human working memory Neuroimage, 5(1), 49-62.

Buchsbaum, B. R., \& D'Esposito, M. (2008). The search for the phonological store: From loop to convolution. Journal of Cognitive Neuroscience, 20(5) $762-778$.

Cabeza, R., Ciaramelli, E., Olson, I. R., \& Moscovitch, M. (2008). The parietal cortex and episodic memory: An attentional account. Nature Reviews Neuroscience, 9(8), 613-625.

Cabeza, R., \& Nyberg, L. (2000). Imaging cognition II: An empirical review of 275 PET and fMRI studies. Journal of Cognitive Neuroscience, 12(1), 1-47.

Carter, C. S., Braver, T. S., Barch, D. M., Botvinick, M. M., Noll, D., \& Cohen, J. D. (1998). Anterior cingulate cortex, error detection, and the online monitoring of performance. Science, 280(5364), 747-749.

Chein, J. M., Ravizza, S. M., \& Fiez, J. A. (2003). Using neuroimaging to evaluate models of working memory and their implications for language processing. Journal of Neurolinguistics, 16(4/5), 315-339.

Chi, R. P., Fregni, F., \& Snyder, A. W. (2010). Visual memory improved by non-invasive brain stimulation. Brain Research, 1353, 168-175.

Clark, C. R., Egan, G. F., McFarlane, A. C., Morris, P., Weber, D., Sonkkilla, C., et al (2000). Updating working memory for words: A PET activation study. Human Brain Mapping, 9(1), 42-54

Cohen, J. D., Perlstein, W. M., Braver, T. S., Nystrom, L. E., Noll, D. C., Jonides, J., et al. (1997). Temporal dynamics of brain activation during a working memory task. Nature, 386(6625), 604-608.

Cohen Kadosh, R., Soskic, S., Iuculano, T., Kanai, R., \& Walsh, V. (2010). Modulating neuronal activity produces specific and long-lasting changes in numerica competence. Current Biology, 20(22), 2016-2020.

Collette, F., Hogge, M., Salmon, E., \& Van der Linden, M. (2006). Exploration of the neural substrates of executive functioning by functional neuroimaging. Neuroscience, 139(1), 209-221.

D’Esposito, M., Aguirre, G. K., Zarahn, E., Ballard, D., Shin, R. K., \& Lease, J. (1998) Functional MRI studies of spatial and nonspatial working memory. Cognitive Brain Research, 7(1), 1-13.

D'Esposito, M. Postle, B. R., Jonides, J., \& Smith, E. E. (1999). The neural substrate and temporal dynamics of interference effects in working memory as revealed by event-related functional MRI. Proceedings of the National Academy of Sciences of the United States of America, 96(13), 7514-7519.

Diana, R. A., Yonelinas, A. P., \& Ranganath, C. (2007). Imaging recollection and familiarity in the medial temporal lobe: A three-component model. Trends in Cognitive Sciences, 11(9), 379-386.

Dobbins, I. G., Simons, J. S., \& Schacter, D. L. (2004). fMRI evidence for separable and lateralized prefrontal memory monitoring processes. Journal of Cognitive Neuroscience, 16(6), 908-920.

Duarte, A., Ranganath, C., \& Knight, R. T.(2005). Effects of unilateral prefrontal lesions on familiarity, recollection, and source memory. Journal of Neuroscience, 25(36) 8333-8337.

Duarte, A., Ranganath, C., Winward, L., Hayward, D., \& Knight, R. T. (2004). Dissociable neural correlates for familiarity and recollection during the encoding and retrieval of pictures. Cognitive Brain Research, 18(3), 255-272.

Elmer, S., Burkard, M., Renz, B., Meyer, M., \& Jancke, L. (2009). Direct current induced short-term modulation of the left dorsolateral prefrontal cortex while learning auditory presented nouns. Behavioral and Brain Functions, 5, 29.

Fecteau, S., Pascual-Leone, A., Zald, D. H., Liguori, P., Theoret, H., Boggio, P. S., et al. (2007). Activation of prefrontal cortex by transcranial direct current stimulation reduces appetite for risk during ambiguous decision making. Journal of Neuroscience, 27(23), 6212-6218.

Ferrucci, R., Marceglia, S., Vergari, M., Cogiamanian, F., Mrakic-Sposta, S., Mameli, F., et al. (2008). Cerebellar transcranial direct current stimulation impairs the practice-dependent proficiency increase in working memory. Journal of Cognitive Neuroscience, 20(9), 1687-1697.
Fiez, J. A., Raife, E. A., Balota, D. A., Schwarz, J. P., Raichle, M. E., \& Petersen, S. E. (1996) A positron emission tomography study of the short-term maintenance of verbal information. Journal of Neuroscience, 16(2), 808-822.

Fregni, F., Boggio, P. S., Nitsche, M., Bermpohl, F., Antal, A., Feredoes, E., et al. (2005) Anodal transcranial direct current stimulation of prefrontal cortex enhances working memory. Experimental Brain Research, 166(1), 23-30.

Gandiga, P. C., Hummel, F. C., \& Cohen, L. G. (2006). Transcranial DC stimulation (tDCS): A tool for double-blind sham-controlled clinical studies in brain stimulation. Clinical Neurophysiology, 117(4), 845-850.

Garavan, H., Kelley, D., Rosen, A., Rao, S. M., \& Stein, E. A. (2000). Practice-related functional activation changes in a working memory task. Microscopy Research and Technique, 51(1), 54-63.

Gevins, A., \& Cutillo, B. (1993). Spatiotemporal dynamics of component processes in human working memory. Electroencephalograpy and Clinical Neurophysiology, $87(3), 128-143$.

Hamidi, M., Tononi, G., \& Postle, B. R. (2008). Evaluating frontal and parietal contributions to spatial working memory with repetitive transcranial magnetic stimulation. Brain Research, 1230, 202-210.

Harbison, J., Atkins, S., \& Dougherty, M. (2011). N-back training task performance: Analysis and model. Proceedings of the Cognitive Science Society 2011.

Hecht, D., Walsh, V., \& Lavidor, M. (2010). Transcranial direct current stimulation facilitates decision making in a probabilistic guessing task. Journal of Neuroscience, 30(12), 4241-4245.

Henson, R. N., Rugg, M. D., Shallice, T., Josephs, O., \& Dolan, R. J. (1999). Recollection and familiarity in recognition memory: An event-related functional magnetic resonance imaging study. Journal of Neuroscience, 19(10), 3962-3972.

Herwig, U., Satrapi, P., \& Schonfeldt-Lecuona, C. (2003). Using the international 10-20 EEG system for positioning of transcranial magnetic stimulation. Brain Topography, 16(2), 95-99.

Honey, G. D., Bullmore, E. T., \& Sharma, T. (2000). Prolonged reaction time to a verbal working memory task predicts increased power of posterior parietal cortical activation. Neuroimage, 12(5), 495-503.

Honey, G. D., Fu, C. H., Kim, J., Brammer, M. J., Croudace, T. J., Suckling, J., et al. (2002). Effects of verbal working memory load on corticocortical connectivity modeled by path analysis of functional magnetic resonance imaging data. Neuroimage, 17(2), 573-582.

Jasper, H. (1958). The ten twenty electrode system of the International Federation. Electroencephalograpy and Clinical Neurophysiology, 10, 371-375.

Jolles, D. D. Grol, M. J., Van Buchem, M. A., Rombouts, S. A. \& Crone, E. A. (2010). Practice effects in the brain: Changes in cerebral activation after working memory practice depend on task demands. Neuroimage, 52(2), 658-668.

Jonides, J., Schumacher, E. H., Smith, E. E., Koeppe, R. A., Awh, E., Reuter-Lorenz, P. A., et al. (1998). The role of parietal cortex in verbal working memory. Journal of Neuroscience, 18(13), 5026-5034.

Jonides, J., Schumacher, E. H., Smith, E. E., Lauber, E. J., Awh, E., Minoshima, S., et al. (1997a). Verbal working memory load affects regional brain activation as measured by PET. Journal of Cognitive Neuroscience, 9, 462-475.

Jonides, J., Schumacher, E. H., Smith, E. E., Lauber, E. J., Awh, E., Minoshima, S., et al. (1997b). Working memory load affects regional brain activation as measured by PET. Journal of Cognitive Neuroscience, 9(4), 462-465.

Jonides, J., Smith, E. E., Marshuetz, C., Koeppe, R. A., \& Reuter-Lorenz, P. A. (1998). Inhibition in verbal working memory revealed by brain activation. Proceedings of the National Academy of Sciences of the United States of America, 95(14), 8410-8413.

Just, M. A., \& Carpenter, P. A. (1992). A capacity theory of comprehension: Individual differences in working memory. Psychological Review, 99(1), 122-149.

Keeser, D., Padberg, F., Reisinger, E., Pogarell, O., Kirsch, V., Palm, U., et al. (2011) Prefrontal direct current stimulation modulates resting EEG and event-related potentials in healthy subjects: A standardized low resolution tomography (sLORETA) study. Neuroimage, 55(2), 644-657.

Kelly, A. M., \& Garavan, H. (2005). Human functional neuroimaging of brain changes associated with practice. Cerebral Cortex, 15(8), 1089-1102.

Kensinger, E. A., Clarke, R. J., \& Corkin, S. (2003). What neural correlates underlie successful encoding and retrieval? A functional magnetic resonance imaging study using a divided attention paradigm. Journal of Neuroscience, 23(6), 2407-2415.

Kirschen, M. P., Chen, S. H., Schraedley-Desmond, P., \& Desmond, J. E. (2005). Loadand practice-dependent increases in cerebro-cerebellar activation in verbal working memory: An fMRI study. Neuroimage, 24(2), 462-472.

Koch, G., Oliveri, M., Torriero, S., Carlesimo, G. A., Turriziani, P., \& Caltagirone, C. (2005). rTMS evidence of different delay and decision processes in a fronto-parietal neuronal network activated during spatial working memory. Neuroimage, 24(1), 34-39.

Koenigs, M., Barbey, A. K., Postle, B. R., \& Grafman, J. (2009). Superior parietal cortex is critical for the manipulation of information in working memory. Journal of Neuroscience, 29(47), 14980-14986.

LaBar, K. S., Gitelman, D. R., Parrish, T. B., \& Mesulam, M. (1999). Neuroanatomic overlap of working memory and spatial attention networks: A functional MRI comparison within subjects. Neuroimage, 10(6), 695-704

Lang, N., Siebner, H. R., Ward, N. S., Lee, L., Nitsche, M. A., Paulus, W., et al. (2005). How does transcranial DC stimulation of the primary motor cortex alter regional neuronal activity in the human brain? European Journal of Neuroscience, 22(2), 495-504.

Lovett, M., Daily, L. Z., \& Reder, L. M. (2000). A source activation theory of working memory: Cross-task prediction of performance in ACT-R. Journal of Cognitive Systems Research, 1, 99-118.

Manoach, D. S., White, N. S., Lindgren, K. A., Heckers, S., Coleman, M. J., Dubal, S., et al. (2004). Hemispheric specialization of the lateral prefrontal cortex for 
strategic processing during spatial and shape working memory. Neuroimage, 21(3), 894-903.

Marshall, L., Molle, M., Siebner, H. R., \& Born, J. (2005). Bifrontal transcranial direct current stimulation slows reaction time in a working memory task. BMC Neuroscience, 6, 23.

Mitchell, K. J., Johnson, M. K., Raye, C. L., \& Greene, E. J. (2004). Prefrontal cortex activity associated with source monitoring in a working memory task. Journal of Cognitive Neuroscience, 16(6), 921-934.

Miyake, A., \& Shah, P. (1999). Models of working memory: Mechanisms of active maintenance and executive control. Cambridge: Cambridge Univeristy Press.

Mottaghy, F. M. (2006). Interfering with working memory in humans. Neuroscience, 139(1), 85-90.

Mottaghy, F. M., Doring, T., Muller-Gartner, H. W., Topper, R., \& Krause, B. J. (2002). Bilateral parieto-frontal network for verbal working memory: An interference approach using repetitive transcranial magnetic stimulation (rTMS). European Journal of Neuroscience, 16(8), 1627-1632.

Mottaghy, F. M., Gangitano, M., Krause, B. J., \& Pascual-Leone, A. (2003). Chronometry of parietal and prefrontal activations in verbal working memory revealed by transcranial magnetic stimulation. Neuroimage, 18(3), 565-575.

Mottaghy, F. M., Pascual-Leone, A., Kemna, L. J., Topper, R., Herzog, H., MullerGartner, H. W., et al. (2003). Modulation of a brain-behavior relationship in verbal working memory by rTMS. Cognitive Brain Research, 15(3), 241-249.

Nitsche, M. A., Cohen, L. G., Wassermann, E. M., Priori, A., Lang, N., Antal, A., et al. (2008). Transcranial direct current stimulation: State of the art 2008. Brain Stimulation, 1(3), 206-223.

Nolde, S. F., Johnson, M. K., \& D'Esposito, M. (1998). Left prefrontal activation during episodic remembering: An event-related fMRI study. Neuroreport, 9(15), 3509-3514.

Nystrom, L. E., Braver, T. S., Sabb, F. W., Delgado, M. R., Noll, D. C., \& Cohen, J. D. (2000). Working memory for letters, shapes, and locations: fMRI evidence against stimulus-based regional organization in human prefrontal cortex. Neuroimage, 11(5 Pt 1), 424-446.

Ohn, S. H., Park, C. I., Yoo, W. K., Ko, M. H., Choi, K. P., Kim, G. M., et al. (2008). Timedependent effect of transcranial direct current stimulation on the enhancement of working memory. Neuroreport, 19(1), 43-47.

Owen, A. M., McMillan, K. M., Laird, A. R., \& Bullmore, E. (2005). N-back working memory paradigm: A meta-analysis of normative functional neuroimaging studies. Human Brain Mapping, 25(1), 46-59.

Penolazzi, B., Di Domenico, A., Marzoli, D., Mammarella, N., Fairfield, B., Franciotti, R., et al. (2010). Effects of transcranial direct current stimulation on episodic memory related to emotional visual stimuli. PLoS One, 5(5), e10623.

Poreisz, C., Boros, K., Antal, A., \& Paulus, W. (2007). Safety aspects of transcranial direct current stimulation concerning healthy subjects and patients. Brain Research Bullettin, 72(4-6), 208-214.

Postle, B. R., Berger, J. S., Goldstein, J. H., Curtis, C. E., \& D’Esposito, M. (2001). Behavioral and neurophysiological correlates of episodic coding, proactive interference, and list length effects in a running span verbal working memory task. Cognitive, Affective, E' Behavioral Neuroscience, 1(1), 10-21.

Postle, B. R., Ferrarelli, F., Hamidi, M., Feredoes, E., Massimini, M., Peterson, M., et al. (2006). Repetitive transcranial magnetic stimulation dissociates working memory manipulation from retention functions in the prefrontal, but not posterior parietal, cortex. Journal of Cognitive Neuroscience, 18(10), $1712-1722$.

Priori, A. (2003). Brain polarization in humans: A reappraisal of an old tool for prolonged non-invasive modulation of brain excitability. Clinical Neurophysiology, 114(4), 589-595.

Ragland, J. D., Turetsky, B. I., Gur, R. C., Gunning-Dixon, F., Turner, T., Schroeder, L. et al.(2002). Working memory for complex figures: An fMRI comparison of letter and fractal n-back tasks. Neuropsychology, 16(3), 370-379.

Salmon, E., Van der Linden, M., Collette, F., Delfiore, G., Maquet, P., Degueldre, C., et al. (1996). Regional brain activity during working memory tasks. Brain, 119(Pt 5), 1617-1625.

Shallice, T. (1988). From neuropsychology to mental structure. Cambridge University Press.

Siebner, H. R., Bergmann, T. O., Bestmann, S., Massimini, M., Johansen-Berg, H. Mochizuki, H., et al. (2009). Consensus paper: Combining transcranial stimulation with neuroimaging. Brain Stimulation, 2(2), 58-80.

Skinner, E. I., \& Fernandes, M. A. (2007). Neural correlates of recollection and familiarity: A review of neuroimaging and patient data. Neuropsychologia, 45(10), 2163-2179.

Smith, E. E., \& Jonides, J. (1998). Neuroimaging analyses of human working memory. Proceedings of the National Academy of Sciences of the United States of America, 95(20), 12061-12068.

Smith, E. E., \& Jonides, J. (1999). Storage and executive processes in the frontal lobes. Science, 283(5408), 1657-1661.

Smith, E. E., Jonides, J., Marshuetz, C., \& Koeppe, R. A. (1998). Components of verbal working memory: Evidence from neuroimaging. Proceedings of the National Academy of Sciences of the United States of America, 95(3), 876-882.

Sporns, O. (2010). Networks of the brain. Cambridge: MIT Press.

Todd, J. J., \& Marois, R. (2004). Capacity limit of visual short-term memory in human posterior parietal cortex. Nature, 428(6984), 751-754.

Utz, K. S., Dimova, V., Oppenlander, K., \& Kerkhoff, G. (2010). Electrified minds: Transcranial direct current stimulation (tDCS) and galvanic vestibular stimulation (GVS) as methods of non-invasive brain stimulation in neuropsychology-A review of current data and future implications. Neuropsychologia, 48(10), 2789-2810.

Venkatakrishnan, A., \& Sandrini, M. (2012). Combining transcranial direct current stimulation and neuroimaging: novel insights in understanding neuroplasticity. Journal of Neurophysiology, 107(1), 1-4.

Vilberg, K. L., \& Rugg, M. D. (2008). Memory retrieval and the parietal cortex: A review of evidence from a dual-process perspective. Neuropsychologia, 46(7), 1787-1799.

Vilberg, K. L., \& Rugg, M. D. (2009). Functional significance of retrieval-related activity in lateral parietal cortex: Evidence from fMRI and ERPs. Human Brain Mapping, 30(5), 1490-1501.

Vines, B. W., Cerruti, C., \& Schlaug, G. (2008). Dual-hemisphere tDCS facilitates greater improvements for healthy subjects' non-dominant hand compared to uni-hemisphere stimulation. BMC Neuroscience, 9, 103.

Xu, Y., \& Chun, M. M. (2006). Dissociable neural mechanisms supporting visual shortterm memory for objects. Nature, 440(7080), 91-95.

Yonelinas, A. P. (2002). The nature of recollection and familiarity: A review of 30 years of research. Journal of Memory and Language, 46, 441-517. 